\title{
A SMART ALGORITHM FOR MULTI-CRITERIA OPTIMIZATION OF MODEL SEQUENCING PROBLEM IN ASSEMBLY LINES
}

\author{
Mudassar RAUF ${ }^{1,4}$, Zailin GUAN ${ }^{1}$, Shoaib SARFRAZ ${ }^{2}$, Jabir MUMTAZ ${ }^{1}$, \\ Essam SHEHAB ${ }^{2,3}$, Mirza Jahanzaib ${ }^{4}$, Muhammad HANIF ${ }^{5}$
}

\begin{abstract}
${ }^{I}$ State Key Lab of Digital Manufacturing Equipment and Technology, School of Mechanical Science and Engineering, Huazhong University of Science and Technology, Wuhan, 430074, P. R. China.

${ }^{2}$ Manufacturing Department, School of Aerospace, Transport and Manufacturing, Cranfield University, UK.

${ }^{3}$ School of Engineering, Nazarbayev University, Astana, Kazakhstan

${ }^{4}$ Faculty of Industrial Engineering, University of Engineering and Technology Taxila, Pakistan

${ }^{5}$ School of Computer Science, Huazhong University of Science and Technology, Wuhan, 430074, P. R. China.
\end{abstract}

\begin{abstract}
Assembly Lines (ALs) are used for mass production as they offer lots of advantages over other production systems in terms of lead time and cost. The advent of mass customization has forced the manufacturing industries to update to Mixed-Model Assembly Lines (MMALs) but at the cost of increased complexity. In the real world, industries need to determine the sequence of models based on various conflicting performance measures/criteria. This paper investigates the Multi-Criteria Model Sequencing Problem (MC-MSP) using a modified simulation integrated Smart Multi-Criteria Nawaz, Enscore, and Ham (SMC-NEH) algorithm. To address the multiple criteria, a modified simulation integrated Smart Multi-Criteria Nawaz, Enscore, and Ham (SMC-NEH) algorithm was developed by integrating a priori approach with NEH algorithm. Discrete Event Simulation (DES) was used to evaluate each solution. A mathematical model was developed for three criteria: flow time, makespan and idle time. Further, to validate the effectiveness of the proposed SMC-NEH a case study and Taillard's benchmark instances were solved and a Multi-Criteria Decision-Making (MCDM) analysis was performed to compare the performance of the proposed SMC-NEH algorithm with the traditional NEH algorithm and its variants. The results showed that the proposed SMC-NEH algorithm outperformed the others in optimizing the conflicting multi-criteria problem.
\end{abstract}

Keywords: Multi-Criteria Optimization, NEH Algorithm, Assembly Lines, Mixed-Model Sequencing, Smart Algorithm 


\section{Introduction}

The concept of Assembly Lines (ALs) has been introduced for mass production as well as cost efficiency. ALs are flow oriented mass production systems where various operations are performed on the products as they move through the system. AL consists of several work stations in series with buffers storage (possibly) in between, where a work station is a group of machines or operators performing one or more operations on the products $[1,2]$. Usually, these workstations are placed in serial, parallel, U-shape and two sided. A transportation system (automatic guided vehicles, conveyor belts or robotic arms) is used to move the product from one workstation to another to perform assembly operations. Each workstation takes some time to perform various operations on the product, this time is known as cycle time [3]. Figure 1 describes a simple mixed-model assembly line with four workstations. It can be seen from Figure 1 that various workstations are located around the conveyor belt and products are moving from one workstation to another in pallets.

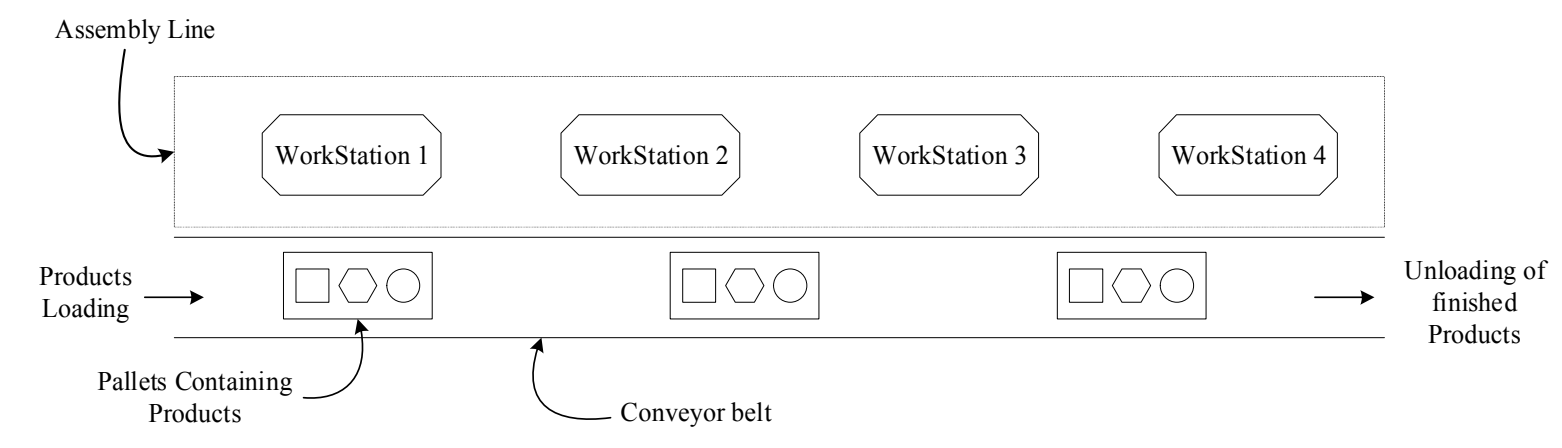

Figure 1 A simple Mixed-Model Assembly Line with four workstations

ALs are classified into three categories - Single Model Assembly Lines (SMALs), Multi Model Assembly Lines (MuMALs) and Mixed Model Assembly Lines (MMALs). MMALs are widely used in mass customization environment. Industries are forced to adopt MMALs because of continuously increasing demand for customized products. MMALs are special types of ALs in which various models of the same products are intermixed to be produced on the same AL with a batch size of one [4]. This naturally solves the batch sizing and inventory problem that exists in MuMAL. Moreover, the investment required for MMALs is quite less than that for separate SMALs but at the cost of increased complexity. However, ineffective use of MMAL results in increased cycle time and higher per unit production cost [5]. There are two Major Challenges in MMALs: Model Sequencing Problem (MSP) and Assembly Line Balancing Problem (ALBP). In ALBP, various tasks/operations are assigned to different stations while optimizing cycle time, balance efficiency and smoothness index etc. However in MSP, sequence/order of various models is determined while optimizing the makespan, flow time, cycle time, idle time and lateness etc $[6,7]$. The MSP with single criteria had been in focus for many years. But in the real world, industries had to face multiple conflicting performance measures/criteria while determining the optimal sequencing. In recent years, researchers have focused on finding the optimal sequence based on various conflicting criteria. Two or more criteria such as idle time, flow time, makespan, earliness, tardiness and their combinations have been used by researchers. In this research, Mixed Model Assembly Line Multi-Criteria Model Sequencing Problem (MMALMC-MSP) is considered with three performance measures: flow time, makespan and idle time. 
Over time various approaches have been developed by researchers for the MultiCriteria Optimization problems (MCO). They are widely divided into three classes. First approach is priori approach in which all the necessary information is given at the start of the decision-making process such as optimization of weighted combination of various criteria or sequential optimization of various criteria [8]. Weighted sum method is the simplest method when all the criteria are to be maximized or minimized. It is generally applied to single dimensional cases as it is difficult to apply on multi-dimensional cases [9]. Further, it uses the linearity of preferences which may not imitate the preferences of the managers and results obtained may not be logical $[10,11]$. Most advanced way in priori approach is the use of MCDM techniques for the ranking of solutions and then selection of the better optimal solution [8]. These techniques can deals with multi-dimensional problems [9]. Second approach is known as posteriori approach in which a better optimal solution is selected from the set of nondominated or Pareto optimal solution [12, 13]. Third approach is called interactive approach in which preferences must be introduced during the solution process and at each step a most preferred solution is selected. Finally, the methodology determined the optimal compromise between the various criteria [14]. Michalos et al. [15] developed a decision making method consists of two stages for multi-criteria assembly line design. In first stage, minimum number of resources required to perform the assembly operations are calculating while in the second stage, assembly was configured to further improve its performance based on multiple criteria. Georgy et al. [16] proposed a model for the evaluation of engineering performance by means of utility function. A multi attribute utility function was developed based on eigenvector prioritization method.

Extensive literature can be found on multi-objective optimization. However, literature on MC-MSP is relatively scarce especially compared to the large number of papers published on single criteria model sequencing problems. Various approaches and models are proposed by researchers to solve the MC-MSP in MMAL environment, namely multi-objective genetic algorithm [17], multi-objective simulated annealing [18], ant colony optimization algorithm [19], and multi-objective artificial bee colony algorithm [20], while considering various performance measures. Comprehensive literature review on MC-MSP has been covered by Yenisey et al. [14] and Minella et al. [21]. Therefore, only the significant and recent research work on MC-MSP in MMAL environment is discussed in the lines that follow. Shao et al. [17] addressed the sequencing problems in pull production systems which were composed of one mixed-model assembly line and one flexible fabrication flow line with limited intermediate buffers. They considered two performance measures simultaneously: minimization of total variation in parts consumption and makespan in the fabrication line. They proposed multiobjective genetic algorithm by integrating the Preto Rank Method (PRM) and proved that the proposed algorithm was more efficient in minimizing both performance measures than existing multi-objective simulated annealing algorithm. Kucukkoc et al. [19] proposed a special mixedmodel assembly line system based on the parallel two-sided assembly line while optimizing the weighted idle time and cycle to cycle work lord smoothness index. They proposed an agentbased ant colony optimization algorithm up to four levels. Salehi et al. [18] proposed a metaheuristic algorithm based on simulated annealing for the optimization of three performance measures: idle cost, setup cost and production rate variation cost. Effectiveness of the proposed algorithm is proved against Lingo software. Fattahi et al. [22] determined the optimal sequence of products considering the feeding lines. They proposed simulated annealing and ant colony heuristic algorithms for optimizing the three performance measures namely: idle time cost, 
workload cost and component deviation cost. Saif et al. [20] considered the simultaneously balancing and sequencing problem while optimizing the workload deviation and total flow time. They introduced Pareto solution in Artificial Bee Colony Algorithm (ABC) and proposed multi-objective ABC algorithm. Norozi et al. [23] proposed intelligence based genetic algorithm for parallel MMALs in which a heuristic method is used for generating the initial population. They optimized work load and makespan. Tahriri et al. [24] proposed a MultiObjective Genetic Algorithm (MOGA) for the simultaneous optimization of three conflicting performance measures: makespan, setup time and cost. Rahimi-vahed [25] proposed a hybrid algorithm based on Particle Swarm Optimization (PSO) and Tabu Search (TS) by integrating Pareto solution for Multi-Objective Optimization (MOO) of MSP. Senthilkumar et al. [26] proposed multi-objective algorithm based on simulated annealing algorithm for level production scheduling. They solved the problem simultaneously for number of step ups and material usage rate. Mansouri et al. [7] proposed a MOGA by integrating the Pareto optimal frontier with general Genetic Algorithm (GA). Marichelvam et al. [27] extended a discrete firefly algorithm for MC-MSP to optimize mean flow time and makespan. Rajendran et al. [28] developed Multi-Objective Ant-Colony algorithm (MOAC) in order to optimize conflicting performance measures - the makespan and total Flowtime. Moghaddam et al. [29] proposed the memetic algorithm for MC-MSP to optimize three different performance measures. Effectiveness of the proposed algorithm was verified against Lingo 6 software. Eren [30] simultaneously minimized the makespan, total completion time, tardiness and earliness while considering the setup time. Integer programming model was proposed to solve MC-MSP for 20 products. However, six Tabu search based methods and one random search method were used for large scale problems. It was concluded that Tabu search based methods are more effective. Allouche et al. [31] proposed a compromise programming and satisfaction based procedure to solve MC-MSP while minimizing makespan, total flow time and total tardiness. From the above literature it can be concluded that various meta-heuristic algorithms have been used for the optimization of conflicting performance measures/criteria with the integration of various MCO approaches.

Beside meta-heuristic algorithms, constructive algorithms were also used by researchers to solve MSP [32]. So far NEH algorithm has been found to be one of the best constructive methods for permutation scheduling and sequencing problems [33]. In recent years various researchers have modified the NEH algorithm to achieve better optimal results. Liu et al. [34] proposed two techniques to improve the performance of NEH algorithm. They developed block properties while integrating the tie breaking rules. These two techniques helped in getting results in a short time. Kalczynski et al. [35] increased the performance of $\mathrm{NEH}$ algorithm by proposing new techniques. It was proved that NEH algorithm is the best algorithm for the makespan minimization problem. They proposed a new priority order with a tie breaking method. Jin et al. [33] proposed block properties method to reduce the complexity and time of the NEH algorithm. Liu et al. [36] proposed a modified NEH algorithm by introducing dispatching rules while prioritizing the models. According to Rossi et al. [37], NEH algorithm is the best constructive algorithm to minimize the makespan. Although it is not the best algorithm for flow time and idle time minimization problems but still it provides significant results. Framinan et al. [38] had adopted NEH algorithm to minimize the flow time, idle time and makespan separately. They proposed various initial sequences for optimization. Minella et al. [39] employed NEH algorithm for flow time optimization as seed in their restarted iterated pareto greedy algorithm. It is acknowledged that the NEH algorithm and its 
variants perform well for single objective problems such as flow time, makespan, idle time and tardiness $[12,14,40]$. But they can also be used for MCO problems. Various methods have been introduced to use NEH for MCO problems. Ding et al. [41] proposed a Multi-Objective NEH (MONEH) for the optimization of both carbon emissions and makespan. They integrated an energy saving process with NEH in which they change the speed of the machine to save energy while keep the makespan unchanged. Arroyo [12] proposed the integration of Pareto solution concept with $\mathrm{NEH}$ to solve the MCO problem. In that research, makespan and tardiness were minimized simultaneously. Liu et al. [40] proposed a hybrid NEH algorithm for multiple criteria. They optimized the makespan and idle time simultaneously by introducing new priority and tie breaking rules. One of the other methods for MCO is the integration of MCDM techniques for the selection of better solutions. MCDM techniques with the integration of NEH algorithm to simultaneously optimize various conflicting performance measures was proposed by the author in a previous studies [42, 43]. Various MCDM techniques have been developed to facilitate the decision maker in the appropriate selection from a pool of solutions. One of the MCDM techniques is Technique for Order of Preference by Similarity to Ideal Solution (TOPSIS). It is used for selecting the best alternative. In TOPSIS, distances to the positive and negative ideal solution are calculated instantaneously to determine the ranking order of all alternatives $[44,45]$. It is a very useful technique for dealing with real world multicriteria decision-making problems. Because of high flexibility and simplicity, it can be easily used in various situation [11, 46, 47]. Therefore, in this work TOPSIS is integrated with the $\mathrm{NEH}$ algorithm for multi-criteria optimization.

Another major problem in MCO is the evaluation of the algorithm's effectiveness based on conflicting objectives. Unlike single criteria optimization, the comparison of an algorithm in MCO environment is quite difficult. A simple comparison method does not exist for such comparisons. Liu et al. [40] and Parthana et al. [44] had used Average Relative Percentage Deviation (ARPD) and MCDM analysis for the comparison of algorithms for MCO while Ding et al. [41] had used coverage metric, distance metrics and distribution spacing for evaluation of the algorithm on the basis of various criteria.

It can be summarized from the above literature that makespan, lateness, flow time, idle and setup time are the important criteria for MSP. Therefore, in this work MC-MSP has been solved while simultaneously optimizing the Flow Time (FT), Makespan (MS) and Idle Time (IDT). The aim to optimize MS is to complete customers' demand as soon as possible while optimization of FT could help to improve the average response time of the system. However, IDT optimization could increase machine utilization. For years, optimization of MS was considered as equivalent to optimization of IDT, but recent research has shown that these are clearly different and can be conflicting [40]. In this research, three conflicting criteria (MS, FT and IDT) are considered for MC-MSP. The consideration of three conflicting criteria has made this problem unique as well as more complex. A modified simulation integrated Smart Multi-Criteria NEH (SMC-NEH) algorithm has been proposed in which TOPSIS, a MCDM technique, has been integrated for decision making. In order to achieve better optimization, a tie breaking rule was also introduced. Further, each solution has been evaluated using Discrete Event Simulation (DES). For multi-criteria optimization of MSP, use of NEH algorithm with the integration of MCDM technique along with the simulation is a novel approach. It provides the algorithm with required decision-making intelligence to solve multi-criteria optimization problems effectively. Proposed SMC-NEH has been applied on a case study of pumps 
assembly lines in which three conflicting criteria (FT, MS and IDT) were simultaneously optimized. To further validate the effectiveness of proposed SMC-NEH, Taillard's benchmark instances were solved and the performance of proposed SMC-NEH was compared with the traditional NEH algorithm and its variants. Evaluation of algorithms' effectiveness for $\mathrm{MCO}$ was performed by ARPD and MCDM analysis.

The rest of the paper is organized as follows. The example problem studied, and mathematical modeling are presented in section 2 while newly proposed algorithm is described in section 3. In section 4, a case study and test cases are presented while results are analyzed and discussed in section 5. Final conclusions and future recommendations are provided in section 6 .

\section{Problem definition}

To meet customers' demand for customized products, industries try to produce various kinds of products with minimum lead time and cost. A major manufacturing problem associated with mixed-model assembly lines scheduling is the MSP. In this problem, optimal sequencing of products/models is determined while considering multiple criteria. This problem can be categorized as Multi-Criteria Model Sequencing Problem (MC-MSP). To improve average response time of the system, complete customers' demand as soon as possible and maximize machine utilization, it is essential to find an optimal compromise between conflicting criteria (FT, MS and IDT). To solve this problem, first step was the development of a mathematical model of the considered criteria. The notations used in this article are given in Table 1.

Table 1 Notations used in this article

\begin{tabular}{ll}
\hline Parameters & \multicolumn{1}{c}{ Description } \\
\hline$n$ & Total number of models/products \\
$i$ & Total number of workstations/machines \\
$j$ & Index for models, $1 \leq i \leq n$ \\
$P T_{i j}$ & Index for workstations, $1 \leq j \leq m$ \\
$A T_{i j}$ & Process time of $i^{\text {th }}$ model at $j^{\text {th }}$ workstation \\
$C T_{i j}$ & Arrival time of $i^{\text {th }}$ model at $j^{\text {th }}$ workstation \\
$W T_{i j}$ & Completion time of $i^{\text {th }}$ model at $j^{\text {th }}$ workstation \\
$I T_{i j}$ & Waiting time of $i^{\text {th }}$ model at $j^{\text {th }}$ workstation \\
$X_{i j}$ & Idle time for $i^{\text {th }}$ model at $j^{\text {th }}$ workstation \\
$a$ & Decision variable with value 1 only if $i^{\text {th }}$ model assigned to $j^{\text {th }}$ \\
$c$ & workstation otherwise zero \\
$l$ & Total number of alternatives \\
$k$ & Total number of Criteria \\
$C V_{l k}$ & Index for alternatives, $1 \leq l \leq a$ \\
$w_{k}$ & Index for criteria, $1 \leq k \leq c$ \\
$N C V_{l k}$ & Value of $k^{\text {th }}$ criteria for $l^{\text {th }}$ alternative \\
$W N C V_{l k}$ & Weight of the $k^{\text {th }}$ criteria \\
\hline & Normalize value of $k^{\text {th }}$ criteria for $l^{\text {th }}$ alternative \\
\hline
\end{tabular}




\begin{tabular}{ll}
\hline$A_{k}^{+}, A_{k}^{-}$ & Set of positive ideal and negative ideal solutions respectively \\
$S_{l}^{+}, S_{l}^{-}$ & Separation measures from positive ideal $\left(A^{+}\right)$and negative ideal \\
& solutions $\left(A^{-}\right)$respectively \\
$C_{l}$ & Relative closeness of alternatives \\
\hline
\end{tabular}

Three conflicting criteria (FT, MS and IDT) for MC-MSP have been illustrated in Equations (1-3).

$$
\text { Min : } \quad F T=\sum_{i=1}^{N}\left(C T_{i}-A T_{i 1}\right)
$$

First criteria for MC-MSP is given in equation (1) and was used to minimize the total flow time.

$$
\text { Min : } \quad M S=\max \left(C T_{i j}\right)
$$

Second criteria for MC-MSP is expressed in equation (2) and was important in minimizing the makespan.

$$
\text { Min : } \quad I D T=\sum_{i=1}^{N} \sum_{j=1}^{M} I T_{i j}
$$

Third criteria is given in equation (3) and was used to minimize the total idle time. Here

$$
I T_{i j}=\left\{\begin{array}{llr}
0 & \text { if } & A T_{i j}-C T_{(i-1) j} \leq 0 \\
A T_{i j}-C T_{(i-1) j} & \text { if } & A T_{i j}-C T_{(i-1) j}>0
\end{array}\right.
$$

Idle time for $i^{\text {th }}$ model at $j^{\text {th }}$ workstation is calculated by equation (4). Idle time is zero if the $i^{\text {th }}$ model arrived at $j^{\text {th }}$ station before the completion of $(i-1)^{\text {th }}$ model.

$$
\sum_{j=1}^{m} X_{i j} \geq 0, \quad \forall\{i=1,2, \ldots, n\}
$$

Equation (5) assured that a workstation can be idle.

$$
\sum_{i=1}^{n} X_{i j}=1, \quad \forall\{j=1,2, \ldots, m\}
$$

Equation (6) indicated that one model can only be assigned to one station.

$$
A T_{11}=0
$$

Equation (7) assured that the arrival time of the first model at first workstation is zero.

$$
A T_{i 1}=C T_{(i-1) 1}
$$

Equation (8) specified that the arrival time of $i^{\text {th }}$ model at first workstation is equal to the completion time of $(i-1)^{\text {th }}$ model. 


$$
A T_{i j}=C T_{i(j-1)} \quad \forall\{j=2,3, \ldots, m\}
$$

Equation (9) indicated that the arrival time of $i^{\text {th }}$ model at $j^{\text {th }}$ workstation is equal to the completion time of $i^{\text {th }}$ model at $(j-1)^{\text {th }}$ workstation. Here $i=1,2, \ldots, n$ and $j=2,3, \ldots, m$.

$$
W T_{1 j}=0 \quad \forall\{j=1,2, \ldots, m\}
$$

Equation (10) showed that the waiting time for first model at all the workstations is zero.

$$
W T_{i 1}=0 \quad \forall\{i=1,2, \ldots, n\}
$$

Equation (11) indicated that the waiting time of the $i^{\text {th }}$ model at first workstation is zero.

$$
W T_{i j}=\left\{\begin{array}{ccc}
0 & \text { if } & A T_{i j}-C T_{(i-1) j} \geq 0 \\
\left|A T_{i j}-C T_{(i-1) j}\right| & \text { if } & A T_{i j}-C T_{(i-1) j}<0
\end{array}\right.
$$

Waiting time of $i^{\text {th }}$ model at $j^{\text {th }}$ workstation can be calculated from equation (12) and it is equal to zero if arrival time $i^{\text {th }}$ model at $j^{\text {th }}$ workstation is greater than the completion time of $(i-1)^{\text {th }}$ model at $j^{\text {th }}$ workstation.

$$
C T_{i j}=A T_{i j}+W T_{i j}+P T_{i j}
$$

Completion time of $i^{\text {th }}$ model at $j^{\text {th }}$ workstation can be calculated from equation (13) and it is equal to the sum of arrival time, waiting time and process time of $i^{\text {th }}$ model at $j^{\text {th }}$ workstation. Following assumptions were used in this paper:

1. MMAL must be balanced before solving MC-MSP

2. The number of various models and their respected process times are known and constant.

3. Movement of the models is asynchronous

4. Travel time of models from one station to another is assumed to be zero.

5. Process time is the sum of set-up time and assembly time.

6. Models Pre-emption is not allowed.

7. Machines are uninterruptedly available and cannot process more than one models at the same time.

\section{Proposed Algorithm (SMC-NEH)}

In proposed SMC-NEH algorithm, decision making technique (TOPSIS) along with DES were integrated with traditional NEH algorithm for multi-criteria optimization.

\subsection{Multi-Criteria Optimization (MCO)}

In a conflicting criteria environment, it is vital to find out the optimal solution which can provide optimal compromise between all the criteria. TOPSIS, a MCDM technique, is suggested for MCO of MC-MSP. It is a very useful technique for dealing with real world multi- 
criteria decision-making problems. Steps to find optimal solution using TOPSIS are given below:

\subsubsection{Decision Matrix}

First step for TOPSIS implementation is the creation of a decision matrix. This consisted of various available alternatives or choices, which are ranked against different objectives or criteria.

\subsubsection{Normalization}

In the second step, Criteria in the decision matrix are normalized using equation (14).

$$
N C V_{l k}=\frac{C V_{l k}}{\sqrt{\sum_{l=1}^{a} C V_{l k}^{2}}}
$$

\subsubsection{Weighted Normalization}

Third step is assignment of weights to the criteria. After weight assignment, weighted normalized value of criteria is calculated using equation (15).

$$
W N C V_{l k}=W_{k} \times N C V_{l k}
$$

\subsubsection{Ideal Solution}

In the fourth step, set of positive ideal $\left(A^{+}\right)$and negative ideal solutions $\left(A^{-}\right)$is determined using equation (16) and (17) respectively for each criteria. In the case of minimization problem, positive ideal $\left(A^{+}\right)$solution is the minimum value of the $k^{\text {th }}$ criteria.

$$
\begin{aligned}
& A_{k}^{+}=\min \left(W N C V_{l k}\right), \quad \forall\{l=1,2,3, \ldots, A\} \\
& A_{k}^{-}=\max \left(W N C V_{l k}\right), \quad \forall\{l=1,2,3, \ldots, A\}
\end{aligned}
$$

\subsubsection{Separation and Closeness}

In the fifth step, separations: positive ideal $\left(A_{k}^{+}\right)$and negative ideal solutions $\left(\overline{A_{k}}\right)$ are calculated using equations (18) and (19) respectively for each alternative. While, Relative closeness to the ideal solution is measured for each alternative using equation (20).

$$
\begin{array}{cc}
S_{l}^{+}=\sqrt{\sum_{k=1}^{C}\left(W N C V_{l k}-W N C V_{k}^{+}\right)^{2}}, & \forall\{l=1,2,3, \ldots, A\} \\
S_{l}^{-}=\sqrt{\sum_{k=1}^{C}\left(W N C V_{l k}-W N C V_{k}^{-}\right)^{2}}, \quad \forall\{l=1,2,3, \ldots, A\} \\
R_{l}=\frac{S_{l}^{-}}{\left(S_{l}^{-}+S_{l}^{+}\right)}
\end{array}
$$




\subsubsection{Ranking}

Last step is the ranking of various alternatives on the bases of relative closeness $\left(R_{l}\right)$. It is the ratio of the distance from negative ideal solution and total distance. The alternative with higher $R_{l}$ value is the better alternative which provides optimal comprise between various conflicting criteria.

\subsection{SMC-NEH:}

NEH algorithm was originally proposed for MSP in Permutation Flow-Shop (PFS) for makespan minimization. Over the past years, researchers have also used it for idle time [40], flow time [38], tardiness [12] and energy minimization [41]. MC-MSP in MMAL is the same as in PFS because of the assumptions considered while defining the problem in Section 2. Consideration of three conflicting criteria has made this problem more complex. In this algorithm, TOPSIS and DES are integrated with NEH algorithm for MCO. To achieve better optimization, a tie breaking rule is also introduced. Based on these, new proposed SMC-NEH is as follows:

1. Sort the products according to the descending sum of process times.

2. Pick the first two and determine the products partial sequence by ranking them on the bases of relative closeness $\left(R_{l}\right)$. To determine the $C V_{l k}$, discrete event simulation is used.

3. Insert the $i^{t h}$ product at the place which provides highest relative closeness $\left(R_{l}\right)$ value. In case of tie, apply tie breaking rule (sequence with minimum idle time is selected) because higher machine utilization reduces production cost.

4. Repeat step 3 until all the products are sequenced.

Flowchart of the proposed SMC-NEH algorithm is given Figure 2 while pseudo code of the algorithm is given in

Table 2. 


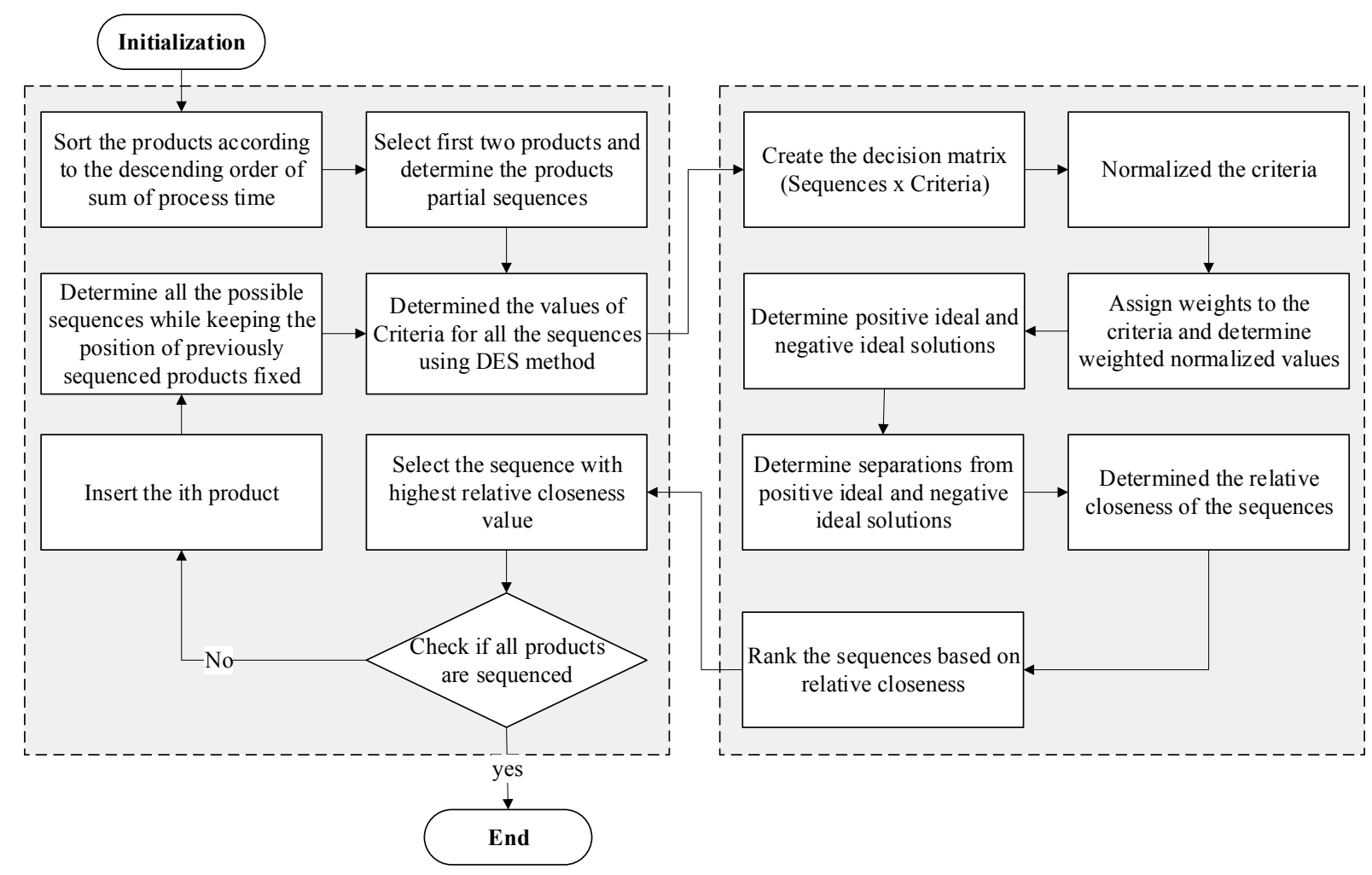

Figure 2 Flowchart of proposed Smart Multi-Criteria Nawaz, Enscore, and Ham (SMC-NEH) algorithm

Table 2 Pseudo code of SMC-NEH algorithm

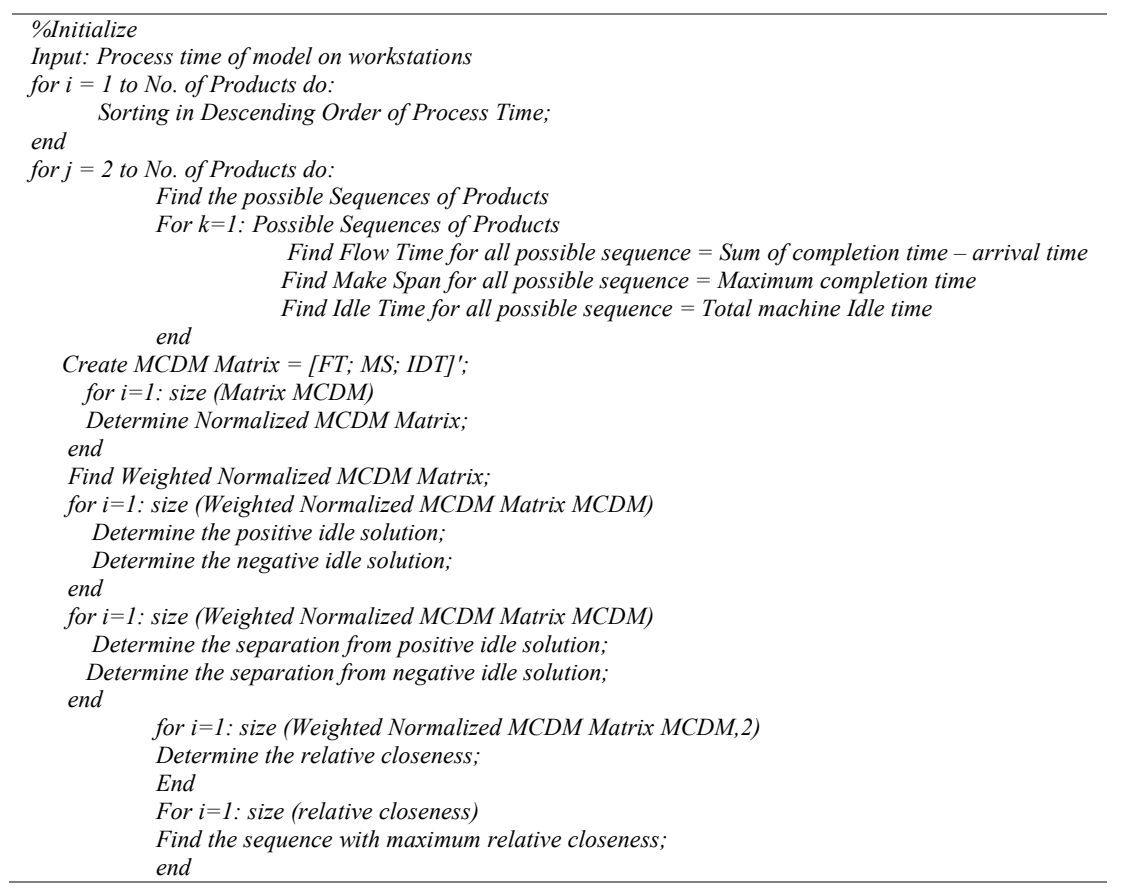

\section{Case Study and Test Problems}

A case study of Mixed model pumps assembly line was conducted to elaborate the proposed SMC-NEH algorithm. Main parts required for assembly of hydraulic pumps are illustrated in Figure 3. Mixed-model pump assembly line is shown in Figure 4. There were thirteen models 
( $i=13$ ) to be assembled on MMAL. Twenty-four operations were required for the assembly of each model on seven workstations $(j=7)$. First station was being used for the assembly of the valve chamber while housing intake, control valve, rare body, safety valve, cam shaft and front body were being assembled on second, third, fourth, fifth, sixth and seventh stations respectively.

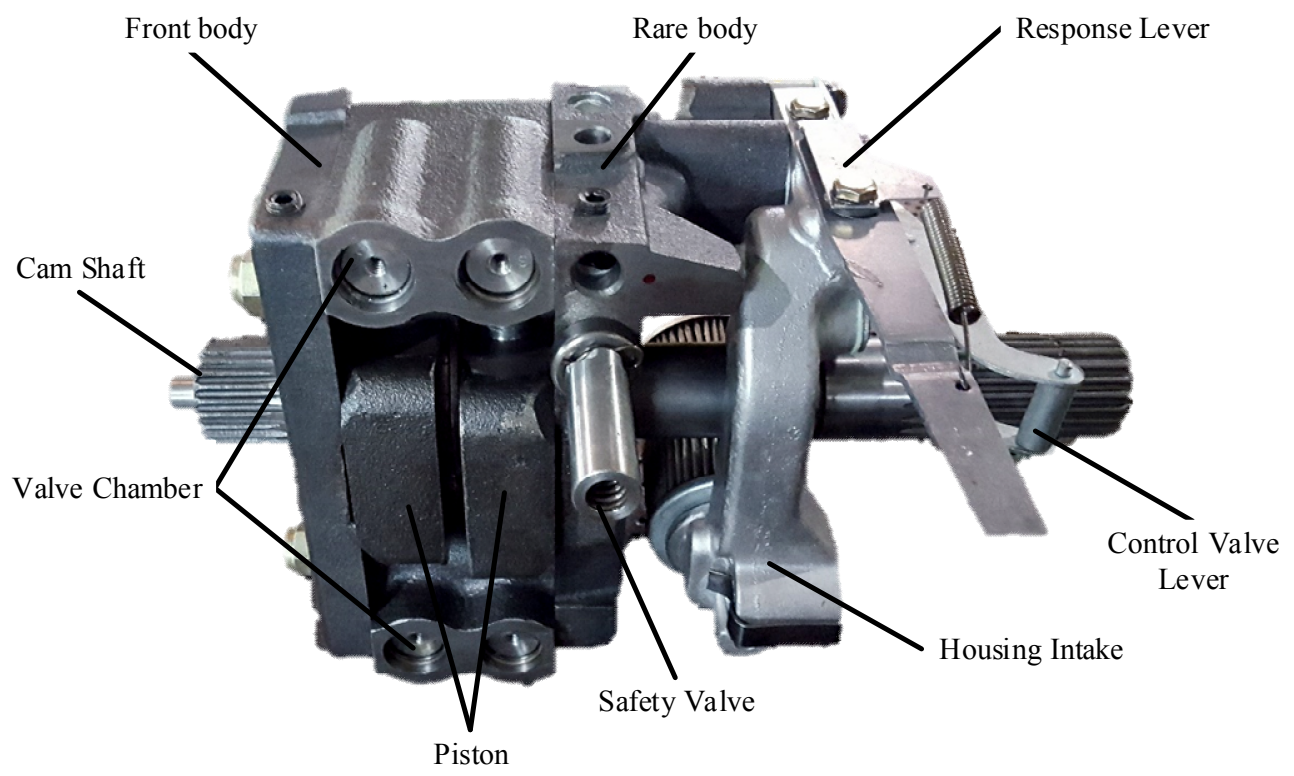

Figure 3 Main parts required for assembly of hydraulic pumps

Semi-automated machines were used to perform the operations. Assembly line under consideration was asynchronous un-paced line. It means an operator starts to work on the next part as soon as it becomes available. Thus, the movement of parts is not coordinated. On completion of service the parts immediately move to the next machine or work station, if the space is available for them. In this MMAL, Models were loaded on workstation 1 and after processing transferred to the next workstation. After passing through various workstations they were unloaded at station 7. Un-paced conveyors were used to transfer the models from one workstation to another.

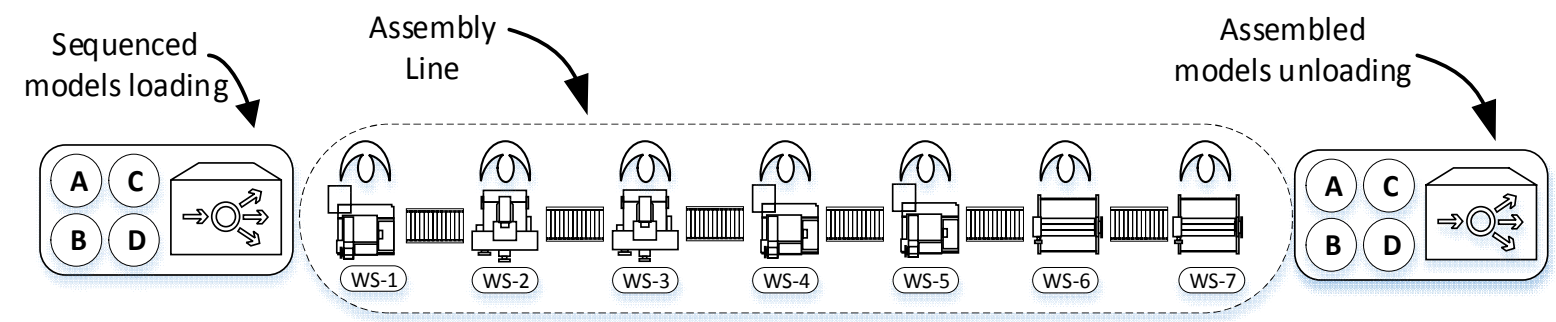

Figure 4 Description of Mixed-Model Pump Assembly line

This study encompasses four weeks of time and motion study to determine the average process time of various models. Time required for each workstation to perform the assembly operation is recorded. As the assembly operations are being performed using semi-automated machines, so process time of operations tends to change because of various factors such as 
fatigue and operator's efficiency etc. Therefore, average process time is calculated to minimize the real-life uncertainty factors. The average process time for each model at various workstations is given in Table 3.

Table 3 Process Time (sec) of Models on Respective Stations

\begin{tabular}{cccccccc}
\hline Model $(i) /$ Station $(j)$ & $\begin{array}{c}\text { Station } \\
(1)\end{array}$ & $\begin{array}{c}\text { Station } \\
(2)\end{array}$ & $\begin{array}{c}\text { Station } \\
(3)\end{array}$ & $\begin{array}{c}\text { Station } \\
(4)\end{array}$ & $\begin{array}{c}\text { Station } \\
(5)\end{array}$ & $\begin{array}{c}\text { Station } \\
(6)\end{array}$ & $\begin{array}{c}\text { Station } \\
(7)\end{array}$ \\
\hline Model (1) & 34.16 & 44.99 & 27.69 & 34.26 & 25.25 & 30.72 & 43.33 \\
Model (2) & 39.38 & 22.51 & 36.15 & 26.42 & 27.33 & 32.47 & 34.90 \\
Model (3) & 32.42 & 32.39 & 26.42 & 27.77 & 30.47 & 23.24 & 31.40 \\
Model (4) & 33.68 & 34.05 & 39.28 & 22.20 & 20.66 & 32.32 & 33.17 \\
Model (5) & 32.31 & 22.40 & 42.00 & 28.12 & 43.16 & 44.39 & 31.02 \\
Model (6) & 32.60 & 35.60 & 25.01 & 41.85 & 31.28 & 39.39 & 32.92 \\
Model (7) & 24.42 & 24.32 & 40.16 & 26.68 & 26.11 & 21.86 & 44.42 \\
Model (8) & 40.65 & 42.12 & 29.10 & 31.70 & 43.76 & 20.00 & 41.48 \\
Model (9) & 29.49 & 32.18 & 35.40 & 26.18 & 27.07 & 27.63 & 37.26 \\
Model (10) & 43.94 & 33.98 & 26.43 & 37.68 & 26.29 & 35.48 & 35.92 \\
Model (11) & 37.81 & 27.36 & 33.85 & 20.10 & 30.62 & 27.09 & 39.69 \\
Model (12) & 26.92 & 42.94 & 29.22 & 42.10 & 26.55 & 43.61 & 27.53 \\
Model (13) & 35.94 & 20.31 & 27.46 & 22.17 & 32.68 & 33.74 & 21.30 \\
\hline
\end{tabular}

It was vital for the company to determine the sequence of models which simultaneously optimized three criteria (flow time, makespan and idle time). Flow time is associated with the response of the assembly line to demand while makespan is used to increase the system throughput. So, minimization of flow time leads to the maximization of makespan and vice versa. Same is the case with machine idle time as minimum idle time significantly increases the flow time and makespan. Therefore, proposed SMC-NEH algorithm was applied for the simultaneous optimization of the above-mentioned conflicting criteria. Optimized sequence obtained from proposed algorithm is $12,7,9,8,6,10,5,4,1,2,3,11,13$ with the FT, MS and IDT values of $3479.88 \mathrm{sec}, 666.75 \mathrm{sec}$ and $121.25 \mathrm{sec}$ respectively.

To further evaluate the performance of the proposed algorithm, Taillard's [48] benchmark instances were used. Taillard had presented 120 instances with 12 different sized problems. These problems ranged from small problems with 20 models and 5 machines to large problems with 500 models and 20 machines. Mainly, these are used for makespan criterion but they have also been used for flow time and idle time [40]. Each problem was solved using the proposed SMC-NEH algorithm, NEH algorithm and its variants for FT and IDT. All algorithms were programmed and run in Matlab ${ }^{\mathrm{TM}} \mathrm{R} 2018 \mathrm{~b}$ on a personal computer with Core I5-5200U, $2.20 \mathrm{GHz}$ with $8.00 \mathrm{~GB}$ memory.

\section{Analysis of Results and Discussions}

In this section, computational results have been briefly described to evaluate the performance of the proposed SMC-NEH for the MCO of FT, MS and IDT in MC-MSP. Unlike the comparison of algorithms for single criteria optimization, comparison of algorithms for $\mathrm{MCO}$ is difficult as algorithms perform differently with respect to various conflicting criteria. Therefore, without the implementation of MCDM analysis, it is not possible to decide which algorithm performed better based on conflicting criteria. So, there was a need for evaluation on the basis of MCDM. Analysis was performed using TOPSIS to select the better algorithm based 
on conflicting criteria. To further evaluate the algorithms' effectiveness, Relative Percentage Deviation (RPD) was also applied where $R P D_{k q}=\left(C V_{k q}-B V_{k q}\right) / B V_{k q}, C V_{k q}$ is the value of criteria $(k)$ obtained by the algorithm for instance $(q)$ and $B V_{k q}$ is the value of criteria $(k)$ obtained by best approach for instance $(q)$. Optimized sequences, RPD values of the criteria and ranking based on TOPSIS for above mentioned algorithms are given in Table 4.

Table 4 Optimized sequence, Average Relative Percentage Deviation (ARPD) and TOPSIS ranking of algorithms

\begin{tabular}{|c|c|c|c|c|c|c|}
\hline Algorithm & Sequence & FT & MS & IDT & Avg. (RPD) & TOPSIS Ranking \\
\hline SMC-NEH & $\begin{array}{c}12,7,9,8,6,10,5,4,1,2 \\
3,11,13\end{array}$ & 0.12 & 0.02 & 0.00 & 0.047 & 90.72 \\
\hline NEH-FT & $\begin{array}{c}5,8,10,2,4,13,3,11,9 \\
6,1,12,7\end{array}$ & 0.00 & 0.07 & 2.44 & 0.84 & 10.08 \\
\hline NEH-MS & $\begin{array}{c}7,3,5,11,9,2,12,8,10 \\
6,4,1,13\end{array}$ & 0.03 & 0.00 & 1.58 & 0.53 & 36.38 \\
\hline NEH-IDT & $\begin{array}{c}12,7,9,8,11,6,5,3,4,1 \\
10,2,13\end{array}$ & 0.13 & 0.02 & 0.00 & 0.05 & 89.78 \\
\hline
\end{tabular}

As it can be seen from Table 4, ARPD value of SMC-NEH is lowest so it can be concluded that proposed SMC-NEH algorithm provides better MCO for conflicting criteria in MC-MSP while NEH-FT is the worst algorithm for MCO. Same is the case of TOPSIS ranking, proposed SMC-NEH algorithm ranked first with 90.72 percentage while NEH-IDT, NEH-MS and NEHFT ranked second, third and fourth respectively. Moreover, the proposed SMC-NEH algorithm simultaneously optimize all three criteria in 0.00186 seconds while NEH-IDT, NEH-MS and NEH-FT provide results in $0.001856,0.001871$ and 0.001869 seconds respectively. Computational Time (CT) difference of proposed SMC-NEH algorithm is insignificant against the compared algorithms while yields better results.

To further validate the effectiveness of the proposed SMC-NEH algorithm, a total of 120 Taillard's instances with 12 different sized problems were solved. For the sake of simplicity, average results of each problem size were compared. ARPD values of algorithms for each problem size are given in Figure 5. It can be seen from Figure 5 that SMC-NEH had the lowest ARPD values while NEH-FT had the highest ARPD Values. From the ARPD results, it can be seen that SMC-NEH provides better optimal compromise between conflicting criteria as compared to other above-mentioned algorithms while NEH-FT provided the worst results. 


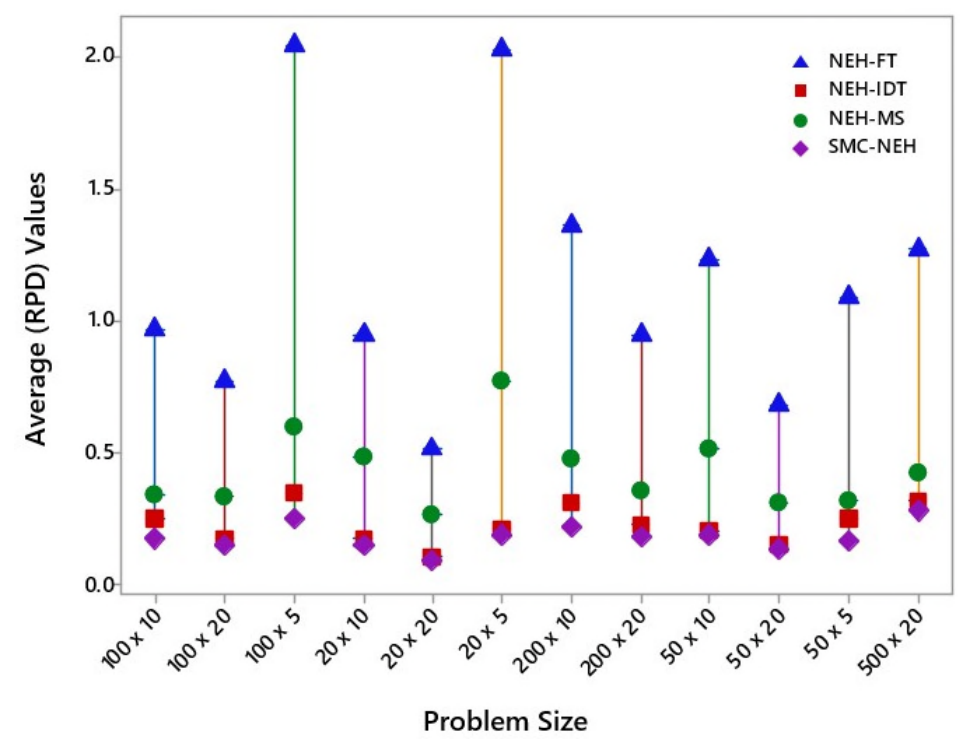

Figure 5 ARPD values of algorithms for each problem size

Further, MCDM analysis was also performed to rank the algorithms based on their effectiveness to optimize multiple conflicting criteria. TOPSIS was used in MCDM analysis. Ranking of algorithms for each problem size based on TOPSIS is given in Figure 6. MCDM analysis also ranked SMC-NEH as the better algorithm for the MCO of MC-MSP while NEHFT ranked as the worst one among other above-mentioned algorithms.

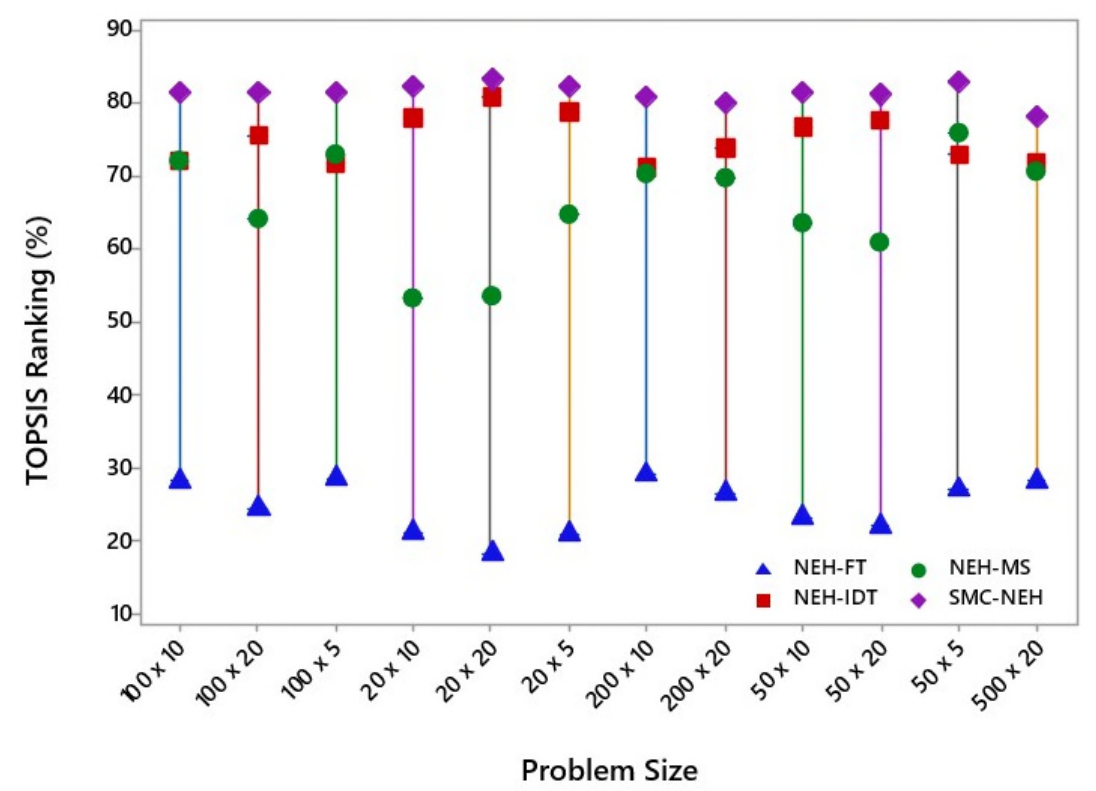

Figure 6 TOPSIS ranking of algorithm for each problem size

RPD values of criteria, ARPD values for each algorithm and TOPSIS ranking of abovementioned algorithms for each problem size is given in Table 5. It can be seen from Table 5 that the ARPD values of criteria for proposed SMC-NEH algorithm was lowest among the other algorithms and the MCDM analysis also ranked proposed SMC-NEH algorithm highest for each problem size. Average of ARPD values of the instances for each algorithm is given in the last row of Table 5 which further strengthens the effectiveness of the proposed algorithm. It can be observed that SMC-NEH had the lowest value of ARPD while NEH-FT had the highest ARPD value and NEH-IDT and NEH-MS are in the second and third place. 
Table 5 Average Relative Percentage Deviation (ARPD) and TOPSIS ranking of algorithms for each problem size

\begin{tabular}{|c|c|c|c|c|c|c|}
\hline \multirow{2}{*}{ Problem Size } & \multirow{2}{*}{ Algorithms } & \multicolumn{3}{|c|}{ RPD } & \multirow{2}{*}{ Avg. (RPD) } & \multirow{2}{*}{ TOPSIS Ranking } \\
\hline & & FT & MS & IDT & & \\
\hline \multirow{4}{*}{$20 \times 5$} & SMC-NEH & 0.41 & 0.11 & 0.03 & 0.19 & 82.08 \\
\hline & NEH-FT & 0.00 & 0.14 & 5.96 & 2.03 & 20.68 \\
\hline & NEH-MS & 0.18 & 0.00 & 2.14 & 0.77 & 64.41 \\
\hline & NEH-IDT & 0.52 & 0.11 & 0.00 & 0.21 & 78.72 \\
\hline \multirow{4}{*}{$20 \times 10$} & SMC-NEH & 0.28 & 0.10 & 0.07 & 0.15 & 82.19 \\
\hline & NEH-FT & 0.00 & 0.12 & 2.72 & 0.94 & 21.06 \\
\hline & NEH-MS & 0.16 & 0.00 & 1.30 & 0.49 & 53.05 \\
\hline & NEH-IDT & 0.38 & 0.13 & 0.00 & 0.17 & 77.73 \\
\hline \multirow{4}{*}{$20 \times 20$} & SMC-NEH & 0.19 & 0.06 & 0.02 & 0.09 & 83.13 \\
\hline & NEH-FT & 0.00 & 0.09 & 1.46 & 0.52 & 18.06 \\
\hline & NEH-MS & 0.09 & 0.00 & 0.69 & 0.26 & 53.36 \\
\hline & NEH-IDT & 0.22 & 0.09 & 0.00 & 0.10 & 80.64 \\
\hline \multirow{4}{*}{$50 \times 5$} & SMC-NEH & 0.38 & 0.05 & 0.07 & 0.16 & 82.81 \\
\hline & NEH-FT & 0.00 & 0.12 & 3.14 & 1.09 & 26.94 \\
\hline & NEH-MS & 0.37 & 0.00 & 0.57 & 0.31 & 75.66 \\
\hline & NEH-IDT & 0.69 & 0.05 & 0.00 & 0.25 & 72.92 \\
\hline \multirow{4}{*}{$50 \times 10$} & SMC-NEH & 0.39 & 0.06 & 0.11 & 0.19 & 81.17 \\
\hline & NEH-FT & 0.00 & 0.14 & 3.58 & 1.24 & 23.13 \\
\hline & NEH-MS & 0.27 & 0.00 & 1.27 & 0.51 & 63.36 \\
\hline & NEH-IDT & 0.53 & 0.07 & 0.00 & 0.20 & 76.60 \\
\hline \multirow{4}{*}{$50 \times 20$} & SMC-NEH & 0.27 & 0.07 & 0.08 & 0.14 & 81.11 \\
\hline & NEH-FT & 0.00 & 0.13 & 1.92 & 0.68 & 21.85 \\
\hline & NEH-MS & 0.19 & 0.00 & 0.74 & 0.31 & 60.86 \\
\hline & NEH-IDT & 0.35 & 0.09 & 0.00 & 0.15 & 77.37 \\
\hline \multirow{4}{*}{$100 \times 5$} & SMC-NEH & 0.58 & 0.04 & 0.13 & 0.25 & 81.27 \\
\hline & NEH-FT & 0.00 & 0.09 & 6.04 & 2.04 & 28.41 \\
\hline & NEH-MS & 0.59 & 0.00 & 1.20 & 0.60 & 72.90 \\
\hline & NEH-IDT & 1.01 & 0.03 & 0.00 & 0.35 & 71.56 \\
\hline \multirow{4}{*}{$100 \times 10$} & SMC-NEH & 0.40 & 0.05 & 0.07 & 0.17 & 81.30 \\
\hline & NEH-FT & 0.00 & 0.12 & 2.79 & 0.97 & 27.99 \\
\hline & NEH-MS & 0.40 & 0.00 & 0.61 & 0.34 & 71.83 \\
\hline & NEH-IDT & 0.70 & 0.05 & 0.00 & 0.25 & 71.87 \\
\hline \multirow{4}{*}{$100 \times 20$} & SMC-NEH & 0.30 & 0.04 & 0.11 & 0.15 & 81.23 \\
\hline & NEH-FT & 0.00 & 0.13 & 2.18 & 0.77 & 24.22 \\
\hline & NEH-MS & 0.28 & 0.00 & 0.73 & 0.34 & 63.92 \\
\hline & NEH-IDT & 0.45 & 0.06 & 0.00 & 0.17 & 75.47 \\
\hline \multirow{4}{*}{$200 \times 10$} & SMC-NEH & 0.53 & 0.03 & 0.10 & 0.22 & 80.61 \\
\hline & NEH-FT & 0.00 & 0.10 & 3.98 & 1.36 & 28.88 \\
\hline & NEH-MS & 0.59 & 0.00 & 0.85 & 0.48 & 70.16 \\
\hline & NEH-IDT & 0.91 & 0.03 & 0.00 & 0.31 & 71.08 \\
\hline \multirow{4}{*}{$200 \times 20$} & SMC-NEH & 0.43 & 0.04 & 0.08 & 0.18 & 79.86 \\
\hline & NEH-FT & 0.00 & 0.13 & 2.71 & 0.95 & 26.20 \\
\hline & NEH-MS & 0.49 & 0.00 & 0.58 & 0.36 & 69.51 \\
\hline & NEH-IDT & 0.63 & 0.05 & 0.00 & 0.23 & 73.64 \\
\hline \multirow{4}{*}{$500 \times 20$} & SMC-NEH & 0.62 & 0.04 & 0.18 & 0.28 & 78.00 \\
\hline & NEH-FT & 0.00 & 0.11 & 3.70 & 1.27 & 28.23 \\
\hline & NEH-MS & 0.83 & 0.00 & 0.44 & 0.42 & 70.41 \\
\hline & NEH-IDT & 0.93 & 0.03 & 0.00 & 0.32 & 71.72 \\
\hline \multirow{4}{*}{ Average } & SMC-NEH & 0.40 & 0.06 & 0.09 & 0.18 & 81.23 \\
\hline & NEH-FT & 0.00 & 0.12 & 3.35 & 1.16 & 24.64 \\
\hline & NEH-MS & 0.37 & 0.00 & 0.93 & 0.43 & 65.79 \\
\hline & NEH-IDT & 0.61 & 0.07 & 0.00 & 0.23 & 74.94 \\
\hline
\end{tabular}

Percentage performance improvement and CT of proposed SMC-NEH algorithm relative to second best algorithm NEH-IDT are given in Table 6. It can be seen from Table 6 that the proposed SMC-NEH achieved $3.31 \%$ to $20.07 \%$ and $0.52 \%$ to $6.36 \%$ better performance in term of ARPD and TOPSIS values respectively for various problem sizes. In case of CT 
consumption, SMC-NEH algorithm consumed $1.89 \%$ to $8.49 \%$ more time as compared to NEH-IDT. This CT difference is insignificant as compared to the percentage performance improvement because any small improvement of above-mentioned criteria can bring significant economic benefits.

Table 6 Percentage performance improvement and CT of proposed SMC-NEH relative to NEH-IDT

\begin{tabular}{cccccccc}
\hline Problem Size & ARPD (\%) & TOPSIS (\%) & CT (\%) & Problem Size & ARPD (\%) & TOPSIS (\%) & CT (\%) \\
\hline $20 \times 5$ & 3.31 & 0.52 & 3.07 & $100 \times 5$ & 16.16 & 6.36 & 4.62 \\
$20 \times 10$ & 7.01 & 2.79 & 3.43 & $100 \times 10$ & 18.37 & 6.16 & 2.87 \\
$20 \times 20$ & 7.27 & 1.52 & 3.00 & $100 \times 20$ & 6.45 & 3.67 & 2.07 \\
$50 \times 5$ & 20.07 & 6.35 & 8.49 & $200 \times 10$ & 17.71 & 6.28 & 2.54 \\
$50 \times 10$ & 3.74 & 2.90 & 3.72 & $200 \times 20$ & 10.70 & 4.05 & 1.89 \\
$50 \times 20$ & 3.46 & 2.36 & 2.91 & $500 \times 20$ & 6.48 & 4.19 & 3.40 \\
\hline
\end{tabular}

Therefore, it can be concluded from the above ARPD and MCDM analysis that SMC-NEH provide a better optimal compromise between conflicting criteria as compared to the traditional $\mathrm{NEH}$ algorithm and its variants for flow time and idle time.

\section{Conclusion}

In this article the SMC-NEH algorithm is proposed by incorporating simulation and TOPSIS for multi-criteria optimization of model sequencing problem in MMAL. In order to validate the performance of the proposed SMC-NEH algorithm, a case study problem of mixed model pumps assembly line and Taillard's benchmark instances (120 instances with 12 different problem sizes) are used. ARPD and MCDM analysis are performed to compare the performance of the proposed SMC-NEH with the traditional NEH algorithm and its variants for flow time and idle time. The ARPD values of criteria for proposed SMC-NEH algorithm is lowest among the other algorithms and the MCDM analysis also ranked proposed SMC-NEH algorithm highest for each problem size while NEH-IDT is ranked second best. As compared to NEH-IDT, proposed SMC-NEH algorithm provided $3.31 \%$ to $20.07 \%$ and $0.52 \%$ to $6.36 \%$ better results in terms of ARPD and TOPSIS values respectively. Results from both the analysis techniques showed that the proposed SMC-NEH algorithm outperforms the rest of the algorithms while NEH-FT is the worst algorithm in optimizing all the conflicting criteria. Thus, it is recommended to use SMC-NEH algorithm for MC-MSP.

This research can be extended by studying the effect of integrating $\mathrm{NEH}$ and its extensions (NEHKK1, NEHKK2, NEH-D, NEHFF and NEHLJP) with various MCDM techniques and Pareto solutions while considering the real-life uncertainties such as deviation in the process time due to human error and machine breakdowns/failures. Additionally, the effectiveness of using MCDM for other MCO problems can be studied in the future.

\section{Acknowledgements}

This work is funded by MOST (Ministry of Science \& Technology of China) the Funds for International Cooperation and Exchange of the National Natural Science Foundation of China (No. 51561125002), the China Postdoctoral Science Foundation (No. 2019M652665 ), the National Natural Science Foundation of China (No. 51275190, 51575211, 51705379, 71620107002, 71131004, 71471071) and, University of Engineering and Technology Taxila, Pakistan. The authors express appreciation to editors, reviewers and Dr. Weibo Liu, National University of Singapore, for their valuable comments and suggestions. 


\section{References}

1. Battaïa, O. and A. Dolgui, A taxonomy of line balancing problems and their solutionapproaches. International Journal of Production Economics, 2013. 142(2): p. 259-277.

2. Chryssolouris, G., Manufacturing systems: theory and practice. 2013: Springer Science \& Business Media.

3. Saif, U., et al., A survey on assembly lines and its types. Frontiers of Mechanical Engineering, 2014. 9(2): p. 95-105.

4. Fattahi, P. and M. Salehi, Sequencing the mixed-model assembly line to minimize the total utility and idle costs with variable launching interval. The International Journal of Advanced Manufacturing Technology, 2009. 45(9-10): p. 987-998.

5. Yagmahan, B., Mixed-model assembly line balancing using a multi-objective ant colony optimization approach. Expert Systems with Applications, 2011. 38(10): p. 12453-12461.

6. Simaria, A.S. and P.M. Vilarinho, A genetic algorithm based approach to the mixed-model assembly line balancing problem of type II. Computers \& Industrial Engineering, 2004. 47(4): p. 391-407.

7. Mansouri, S.A., A multi-objective genetic algorithm for mixed-model sequencing on JIT assembly lines. European Journal of Operational Research, 2005. 167(3): p. 696-716.

8. Deb, K., et al., An interactive evolutionary multiobjective optimization method based on progressively approximated value functions. IEEE Transactions on Evolutionary Computation, 2010. 14(5): p. 723-739.

9. Triantaphyllou, E., Multi-criteria decision making methods, in Multi-criteria decision making methods: A comparative study. 2000, Springer. p. 5-21.

10. Ishizaka, A. and P. Nemery, Multi-criteria decision analysis: methods and software. 2013: John Wiley \& Sons.

11. Velasquez, M. and P.T. Hester, An analysis of multi-criteria decision making methods. International Journal of Operations Research, 2013. 10(2): p. 56-66.

12. Arroyo, J. and V. Armentano, A partial enumeration heuristic for multi-objective flowshop scheduling problems. Journal of the Operational Research Society, 2004. 55(9): p. 1000-1007.

13. Ciavotta, M., G. Minella, and R. Ruiz, Multi-objective sequence dependent setup times permutation flowshop: A new algorithm and a comprehensive study. European Journal of Operational Research, 2013. 227(2): p. 301-313.

14. Yenisey, M.M. and B. Yagmahan, Multi-objective permutation flow shop scheduling problem: Literature review, classification and current trends. Omega, 2014. 45: p. 119-135.

15. Michalos, G., et al., Multi criteria assembly line design and configuration-An automotive case study. 2015. 9: p. 69-87.

16. Georgy, M.E., et al., Utility-function model for engineering performance assessment. 2005. 131(5): p. 558-568.

17. Shao, X., et al., Metaheuristic approaches to sequencing mixed-model fabrication/assembly systems with two objectives. The International Journal of Advanced Manufacturing Technology, 2010. 48(9-12): p. 1159-1171.

18. Salehi, M., et al., Multi-criteria sequencing problem in mixed-model synchronous assembly lines. The International Journal of Advanced Manufacturing Technology, 2013. 67(1-4): p. 983993.

19. Kucukkoc, I. and D.Z. Zhang, Simultaneous balancing and sequencing of mixed-model parallel two-sided assembly lines. International Journal of Production Research, 2014. 52(12): p. 36653687.

20. Saif, U., et al., Multi-objective artificial bee colony algorithm for simultaneous sequencing and balancing of mixed model assembly line. The International Journal of Advanced Manufacturing Technology, 2014. 75(9-12): p. 1809-1827.

21. Minella, G., R. Ruiz, and M. Ciavotta, A review and evaluation of multiobjective algorithms for the flowshop scheduling problem. INFORMS Journal on Computing, 2008. 20(3): p. 451-471. 
22. Fattahi, P., et al., Sequencing mixed-model assembly lines by considering feeding lines. The International Journal of Advanced Manufacturing Technology, 2012. 61(5-8): p. 677-690.

23. Norozi, A., M. Ariffin, and N. Ismail, Application of intelligence based genetic algorithm for job sequencing problem on parallel mixed-model assembly line. The American Journal of Engineering and Applied Sciences, 2010. 3(1): p. 831-840.

24. Tahriri, F., S. Zawiah Md Dawal, and Z. Taha, Multiobjective fuzzy mixed assembly line sequencing optimization model. Journal of Applied Mathematics, 2014. 2014.

25. Rahimi-Vahed, A., S. Mirghorbani, and M. Rabbani, A hybrid multi-objective particle swarm algorithm for a mixed-model assembly line sequencing problem. Engineering Optimization, 2007. 39(8): p. 877-898.

26. Ramalingam, S. and R.A. Subramanian, Solving Level Scheduling in Mixed Model Assembly Line by Simulated Annealing Method. Circuits and Systems, 2016. 7(06): p. 907.

27. Marichelvam, M.K., T. Prabaharan, and X.S. Yang, A discrete firefly algorithm for the multiobjective hybrid flowshop scheduling problems. IEEE transactions on evolutionary computation, 2014. 18(2): p. 301-305.

28. Rajendran, C. and H. Ziegler, A multi-objective ant-colony algorithm for permutation flowshop scheduling to minimize the makespan and total flowtime of jobs, in Computational Intelligence in Flow Shop and Job Shop Scheduling. 2009, Springer. p. 53-99.

29. Tavakkoli-Moghaddam, R. and A. Rahimi-Vahed, Multi-criteria sequencing problem for a mixed-model assembly line in a JIT production system. Applied Mathematics and Computation, 2006. 181(2): p. 1471-1481.

30. Eren, T., A multicriteria flowshop scheduling problem with setup times. Journal of Materials Processing Technology, 2007. 186(1-3): p. 60-65.

31. Allouche, M.A., et al., Solving multi-criteria scheduling flow shop problem through compromise programming and satisfaction functions. European journal of operational research, 2009. 192(2): p. 460-467.

32. Nawaz, M., E.E. Enscore Jr, and I. Ham, A heuristic algorithm for the m-machine, $n$-job flowshop sequencing problem. Omega, 1983. 11(1): p. 91-95.

33. Jin, F., S. Song, and C. Wu, An improved version of the NEH algorithm and its application to large-scale flow-shop scheduling problems. IIE Transactions, 2007. 39(2): p. 229-234.

34. Liu, G., S. Song, and C. Wu. Two techniques to improve the NEH algorithm for flow-shop scheduling problems. in International Conference on Intelligent Computing. 2011. Springer.

35. Kalczynski, P.J. and J. Kamburowski, An improved NEH heuristic to minimize makespan in permutation flow shops. Computers \& Operations Research, 2008. 35(9): p. 3001-3008.

36. Liu, G., S. Song, and C. Wu, Some heuristics for no-wait flowshops with total tardiness criterion. Computers \& operations research, 2013. 40(2): p. 521-525.

37. Rossi, F.L., M.S. Nagano, and R.F.T. Neto, Evaluation of high performance constructive heuristics for the flow shop with makespan minimization. The International Journal of Advanced Manufacturing Technology, 2016. 87(1-4): p. 125-136.

38. Framinan, J., R. Leisten, and C. Rajendran, Different initial sequences for the heuristic of Nawaz, Enscore and Ham to minimize makespan, idletime or flowtime in the static permutation flowshop sequencing problem. International Journal of Production Research, 2003. 41(1): p. 121-148.

39. Minella, G., R. Ruiz, and M. Ciavotta, Restarted Iterated Pareto Greedy algorithm for multiobjective flowshop scheduling problems. Computers \& Operations Research, 2011. 38(11): p. 1521-1533.

40. Liu, W., Y. Jin, and M. Price, A new Nawaz-Enscore-Ham-based heuristic for permutation flowshop problems with bicriteria of makespan and machine idle time. Engineering Optimization, 2016. 48(10): p. 1808-1822.

41. Ding, J.-Y., S. Song, and C. Wu, Carbon-efficient scheduling of flow shops by multi-objective optimization. European Journal of Operational Research, 2016. 248(3): p. 758-771. 
42. Rauf, M., Sarfraz, S., Shehab, E., Javeed, S. and Jahanzaib, M.,. Multi-Objective Optimization for Models Sequencing in Mixed-Model Assembly Lines. in Advances in Manufacturing Technology XXXI: Proceedings of the 15th International Conference on Manufacturing Research, Incorporating the 32nd National Conference on Manufacturing Research, September 5-7, 2017, University of Greenwich, UK. 2017. IOS Press.

43. Rauf, M., M. Jahanzaib, and M.H. Aziz, Modified NEH Algorithm for Multi-Objective Sequencing in Mixed-Model Assembly Lines. Mehran University Research Journal of Engineering and Technology, 2018. 37(3): p. 545-552.

44. Parthanadee, P. and J. Buddhakulsomsiri, Simulation modeling and analysis for production scheduling using real-time dispatching rules: A case study in canned fruit industry. Computers and Electronics in Agriculture, 2010. 70(1): p. 245-255.

45. Peng, C., H. Du, and T.W. Liao, A research on the cutting database system based on machining features and TOPSIS. Robotics and Computer-Integrated Manufacturing, 2017. 43: p. 96-104.

46. RAUF, M., et al. Multi-Criteria Inventory Classification Based on Multi-Criteria Decision-Making (MCDM) Technique. in Advances in Manufacturing Technology XXXII: Proceedings of the 16th International Conference on Manufacturing Research, incorporating the 33rd National Conference on Manufacturing Research, September 11-13, 2018, University of Skövde, Sweden. 2018. IOS Press.

47. iç, Y.T., An experimental design approach using TOPSIS method for the selection of computerintegrated manufacturing technologies. Robotics and Computer-Integrated Manufacturing, 2012. 28(2): p. 245-256.

48. Taillard, E., Benchmarks for basic scheduling problems. european journal of operational research, 1993. 64(2): p. 278-285. 
2019-07-31

\title{
A smart algorithm for multi-criteria optimization of model sequencing problem in assembly lines
}

\author{
Rauf, Mudassar
}

Elsevier

Rauf M, Guan Z, Sarfraz S, et al., (2020) A smart algorithm for multi-criteria optimization of model sequencing problem in assembly lines. Robotics and Computer-Integrated

Manufacturing, Volume 61, February 2020, Article number 101844

https://doi.org/10.1016/j.rcim.2019.101844

Downloaded from Cranfield Library Services E-Repository 